\title{
Application Research on Energy Saving Technology of Waste Heat Recovery and Defrosting for Low Temperature Cold Storage
}

\author{
Li Guangpeng ${ }^{1,2 *}$, Zhou Yanrui ${ }^{1}$ and Sun Liwen ${ }^{1}$ \\ ${ }^{1}$ Shandong Institute of Commerce \& Technology, Jinan, Shandong 250103, China \\ ${ }^{2}$ School of Mines, China University of Mining and Technology, Xuzhou, China
}

\begin{abstract}
In this paper, a glycol defrosting system using waste heat exhaust from refrigeration compressor is designed. The defrosting numerical model, heat transfer model and frost growth model were established. The frost thickness, defrosting heat consumption and ethylene glycol flow rate were calculated, and the ethylene glycol pipeline was designed. The project solves the problems of traditional defrosting of cold storage, such as high power consumption, fluctuation of storage temperature, hidden danger of safety, high condensation temperature and low efficiency of refrigeration system.
\end{abstract}

\section{Introduction}

As a low temperature refrigeration equipment, cold storage is widely used in cold chain logistics equipment[1], which is the key to ensure the storage quality of food and medicine. In the cold storage, when the surface temperature of the cooler heat exchanger is lower than the dew point temperature, the surface of the heat exchanger will form condensation, and frost will be formed when the temperature is lower than the freezing point of water[2]. With the increase of time, the whole surface of the heat exchanger will form a dense frost layer[3]. Due to the low thermal conductivity of the frost layer and the large flow resistance of the air, the heat transfer resistance of the heat exchanger increases and the heat transfer coefficient decreases, which greatly reduces the performance of the heat exchanger and increases the energy consumption of the system. Defrost is necessary to improve the performance of the system.

This paper intends to use the refrigeration compressor to exhaust waste heat and develop the ethylene glycol defrosting system in view of the problems such as high power consumption, fluctuation of storage temperature, safety risks, high condensing temperature and low efficiency of refrigeration system in the traditional defrosting of cold storage. The project mainly solves the key technical problems of system matching and coordination, and carries out practical application verification, in order to obtain a safer and more efficient energy-saving cold storage system.

\section{Principle of hot refrigerant defrosting cycle}

Hot refrigerant defrost is to introduce the refrigerant steam discharged from the compressor into the evaporator, and melt the frost layer on the surface of the evaporator by using the heat released when the superheated steam condenses. The original accumulated ammonia liquid and lubricating oil in the evaporator are discharged into the defrosting drain bucket or low pressure circulation reservoir under the action of pressure difference[4]. 


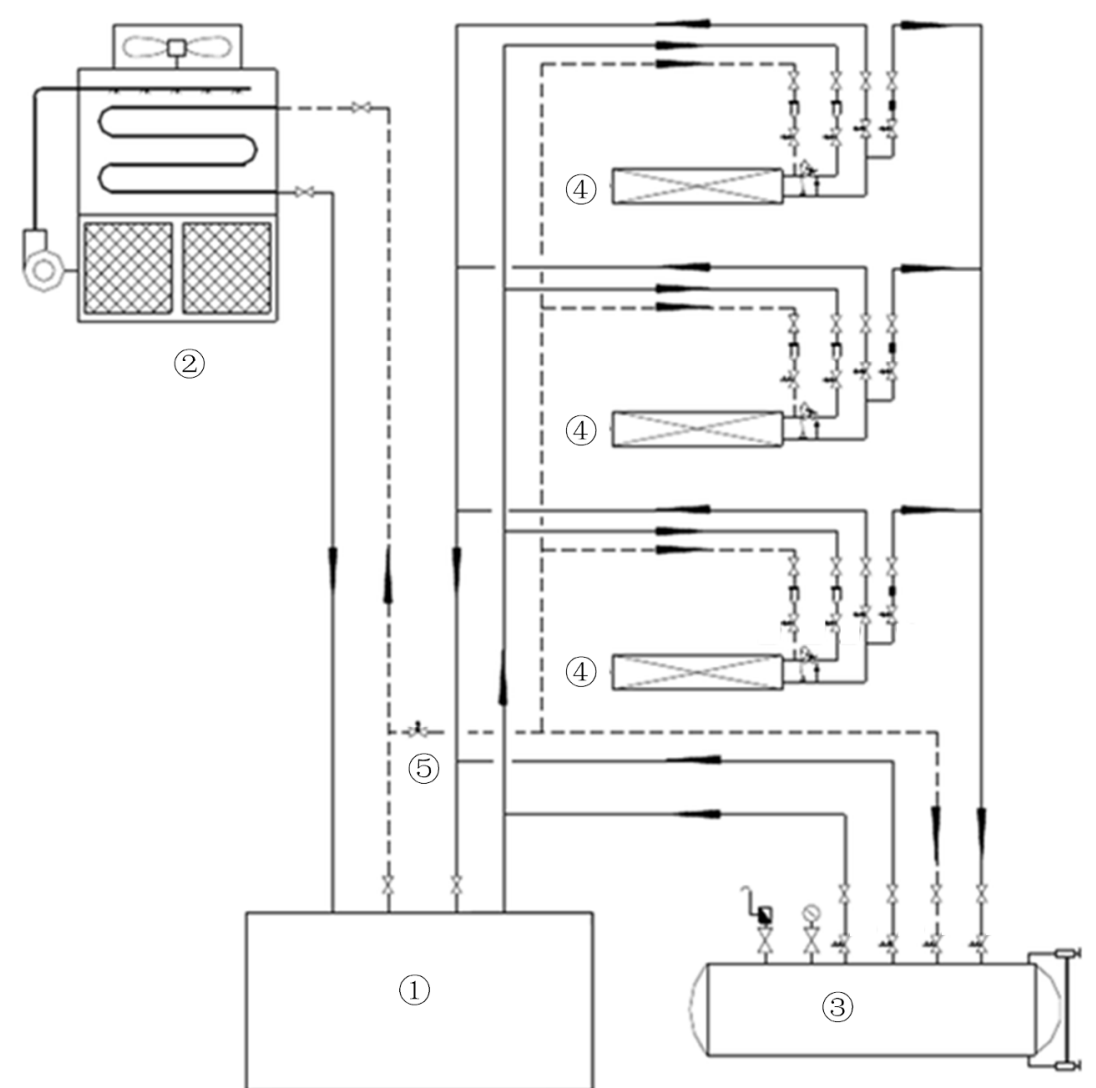

Figure 1. Principle of hot refrigerant defrosting cycle.

1- Compressor 2- Evaporative condenser 3- Circulating drum 4-Air cooler 5- Defrost stop valve

\section{Ethylene glycol solution heat recovery defrosting device}

\subsection{Establish mathematical model, determine air circulation, wet bulb temperature, and calculate frosting amount}

The air cooler is a kind of evaporator used to cool the air. All kinds of aluminum or copper fins are often installed on the air side to increase the area of the heat exchanger. It has the advantages of compact structure and good heat transfer performance, so it is widely used in refrigeration equipment. The object studied in this paper is the air cooler used in cold storage[5]. Its structural parameters are as follows: the fin thickness is $0.35 \mathrm{~mm}$, the fin spacing is $4 \mathrm{~mm}$, the total number of fins is 288 , the number of tube rows is 5 rows, the number of branches is 6 , the number of main pipes is 72 , and the spacing of tubes perpendicular to the direction of air flow is $33 \mathrm{~mm}$. The fan has two, the diameter of the blade is $530 \mathrm{~mm}$, the motor voltage is $380 \mathrm{~V}$, the power is $170 \mathrm{~W}$.

\subsubsection{Numerical model of frosting in finned tube heat exchangers}

Figure 2 shows the finned tube heat exchanger structure, which has a copper tube jacket and aluminum fins to maintain a relatively low temperature as a cold body, through the cooling air flow of relatively high temperature and high relative humidity to provide heat.

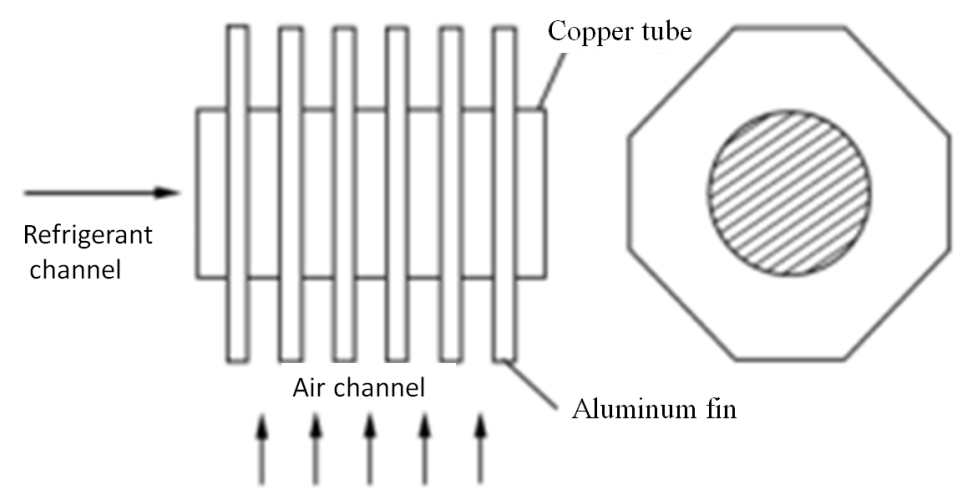

Figure 2. Principle of hot refrigerant defrosting cycle. 
The finned surface frost model is a one-dimensional transient model, which is used to describe the energy and mass migration within the frost layer. The basic governing equations and boundary conditions are described below.

Vapor diffusion equation:

$$
\frac{\partial\left(\varphi_{a} \rho_{v}\right)}{\partial t}-m=\frac{\partial}{\partial z}\left(D_{e f f} \frac{\partial \rho_{v}}{\partial z}\right)
$$

Here, $D_{\text {eff }}$ is the actual vapor diffusion coefficient inside the frost layer $(\mathrm{m} / \mathrm{s})$.

Energy conservation equation:

$$
\rho_{f} c_{p} \frac{\partial y}{\partial x}+\mathrm{m} h_{s g}=\frac{\partial}{\partial z}\left[k_{e f f} \frac{\partial T}{\partial z}\right]
$$

Frost continuity equation:

$$
\frac{\partial \varepsilon_{\beta}}{\partial \mathrm{t}}+\frac{m}{\rho_{i}}=0
$$

The mass transfer boundary condition is

$$
h_{m f}\left[W_{0}-W_{\left(z=\delta_{f, t}\right)}\right]=D_{e f f, s} \frac{\partial \rho_{v\left(z=\delta_{f, t}\right)}}{\partial Z}+\rho_{f} \frac{d \delta_{f}}{d t}
$$

Where, $\mathrm{D}_{\text {eff, s }}$ is the actual vapor diffusion coefficient at the frost layer interface $(\mathrm{m} / \mathrm{s})$.

The thermal conductivity boundary condition is

$$
\begin{gathered}
h_{f}\left[T_{0}-T_{\left(z=\delta_{f, t}\right)}\right]=K_{e f f} \frac{\partial T_{\left(z=\delta_{f, t}\right)}}{\partial z}-h_{s g} \rho_{f} \frac{d \delta_{f}}{d t}(t) \\
\mathrm{T}_{(\mathrm{z}=0, \mathrm{t})}=\mathrm{T}_{\mathrm{c}(\mathrm{x}, \mathrm{y})}
\end{gathered}
$$

Where, $\mathrm{T}_{\mathrm{C}}$ is the fin surface temperature, $\mathrm{T}_{0}$ and $\mathrm{W}_{0}$ are the average airflow temperature and humidity in the fin area.

According to the conservation equation of mass and energy in the fin region:

$$
\begin{aligned}
& \rho_{\mathrm{a}} \mathrm{Q}\left(\mathrm{c}_{\mathrm{pa}} \Delta \mathrm{T}+2500 \Delta \mathrm{W}\right)=\mathrm{q}_{\text {total }} \\
& \rho_{\mathrm{a}} \mathrm{Q} \Delta \mathrm{W}=\int \mathrm{h}_{\mathrm{mf}} \rho_{\mathrm{a}}\left(\mathrm{W}_{0}-\mathrm{W}_{\mathrm{fs}}\right) \mathrm{dA}
\end{aligned}
$$

\subsubsection{Heat transfer model}

Along the direction of air flow, the evaporator is divided into each control body according to the tube row. Each row of tubes and its fins are taken as a control unit, and the air outlet state of the previous control unit is taken as the air inlet state of the next control unit, as shown in Fig.3. After dividing the control body in this way, the model can consider the influence of heat exchanger structure, frost layer thickness and wet air state change along the passage.

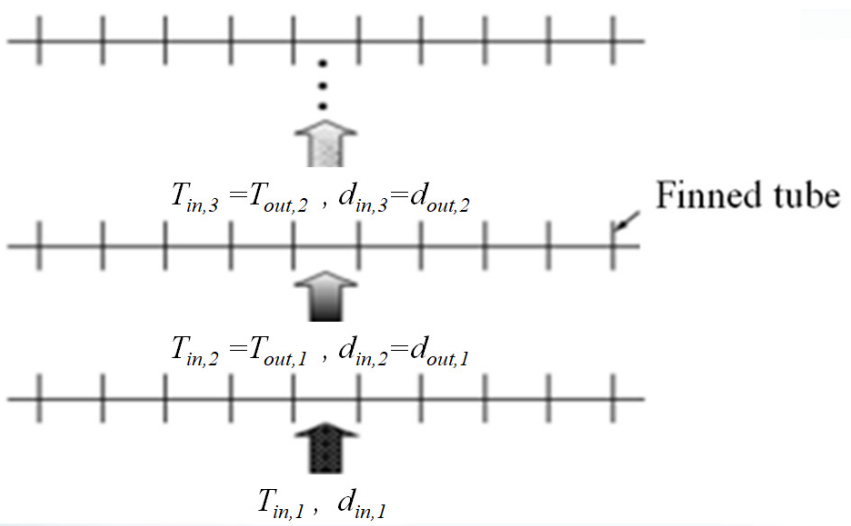

Figure 3. Heat transfer model of finned tube.

According to the conservation equation of mass and energy in the fin region:

$$
\begin{aligned}
& \rho_{\mathrm{a}} \mathrm{Q}\left(\mathrm{c}_{\mathrm{pa}} \Delta \mathrm{T}+2500 \Delta \mathrm{W}\right)=\mathrm{q}_{\text {total }} \\
& \rho_{\mathrm{a}} \mathrm{Q} \Delta \mathrm{W}=\int \mathrm{h}_{\mathrm{mf}} \rho_{\mathrm{a}}\left(\mathrm{W}_{0}-\mathrm{W}_{\mathrm{fs}}\right) \mathrm{dA}
\end{aligned}
$$

and

$$
\begin{gathered}
\Delta \mathrm{T}=\mathrm{T}_{\text {in }}+\mathrm{T}_{\text {out }} \\
\Delta \mathrm{W}=\mathrm{W}_{\text {in }}+\mathrm{W}_{\text {out }}
\end{gathered}
$$

\subsubsection{Frost growth model}

$$
\mathrm{q}_{\mathrm{m}, \mathrm{fr}}=\mathrm{h}_{\mathrm{m}} \mathrm{A}_{\mathrm{T}}\left(\mathrm{d}_{\mathrm{a}, \mathrm{i}}-\mathrm{d}_{\mathrm{fr}}\right)=\mathrm{q}_{\mathrm{m}, \rho}+\mathrm{q}_{\mathrm{m}, \delta}
$$

$$
\mathrm{q}_{\mathrm{m}, \rho}=\mathrm{A}_{\mathrm{T}} \mathrm{D}_{\mathrm{S}}\left(\frac{1-\rho_{\mathrm{fr}} / \rho_{\text {ice }}}{1+\left(\rho_{\mathrm{fr}} / \rho_{\text {ice }}\right)^{0.5}}\right)\left(\frac{\mathrm{d} \rho_{\mathrm{v}}}{\mathrm{dx}}\right)_{\mathrm{s}}
$$

Energy balance form is:

$$
\mathrm{Q}=\mathrm{A}_{\mathrm{T}} \mathrm{D}_{\mathrm{S}} \frac{\mathrm{dT} \mathrm{V}}{\mathrm{dx}}+\mathrm{d}_{\mathrm{fr}} \rho \mathrm{h}_{\mathrm{fr}}
$$

According to the above mathematical model and boundary conditions, it is assumed that in common working conditions of cold storage air cooler, that is, the evaporation temperature of cold storage is $-30^{\circ} \mathrm{C}$, the air inlet temperature of cold air cooler is $-18^{\circ} \mathrm{C}$, the relative humidity in the cold storage is $60 \%$, and the air inlet velocity is $3 \mathrm{~m} / \mathrm{s}$. It is calculated that after four hours of cumulative operation of the compressor, the frost thickness is $3 \mathrm{~mm}$ and the frosting amount is $2000 \mathrm{~g}$. 


\subsection{The calculation method is used to determine the heat consumption of defrosting and the circulating amount of ethylene glycol}

The total heat input in evaporator defrosting $\mathrm{Q}=\mathrm{Q} 1+\mathrm{Q} 2+\mathrm{Q} 3+\mathrm{Q} 4$, where $\mathrm{Q} 1$ is the heat required for the frost layer to dissolve into water at $0^{\circ} \mathrm{C}, \mathrm{Q} 2$ is the heating heat of evaporator, water plate and metal frame, Q3 is the heat absorption of the residual refrigerant working medium in the evaporator coil tube, Q4 is the heat added to the surroundings.

In order to achieve good defrosting effect and the purpose of energy saving, Q1 is necessary, the smaller the value of Q2, Q3 and Q4, the better the energy saving effect. This is because when the warehouse is put into normal operation, this part of the heat must consume cooling capacity to offset, which will pay unnecessary energy consumption. In order to reduce this additional energy consumption, a series of corresponding measures can be taken. There are two measures taken in this paper, one of which is the return air delay. After the defrosting instruction is issued, the liquid inlet solenoid valve of the evaporator is closed, but the return air solenoid valve is delayed and closed to reduce the refrigerant working medium remaining in the evaporator. The other is to stop running the forward fan when defrosting to reduce the heat added to the surrounding environment.

The melting heat of frost is

$$
Q_{1}=G_{f} \times\left[C_{p f}\left(0-t_{f}\right)+r_{f}\right]=775 \mathrm{~kJ}
$$

Where, $\mathrm{G}_{\mathrm{f}}$ is the weight of the frost layer to be melted, $2 \mathrm{~kg} . \mathrm{C}_{\mathrm{pf}}$ is the average specific heat of frost layer, $2050 \mathrm{~J} / \mathrm{kg} . \mathrm{K}) . \mathrm{T}_{\mathrm{f}}$ is the average temperature of the frost layer, which is $-25^{\circ} \mathrm{C}$ between the evaporation temperature and the cold storage temperature according to the situation. $\mathrm{R}_{\mathrm{f}}$ is the melting heat of the frost $3.36 \times 105 \mathrm{~J} / \mathrm{kg}$.

The total heat increased by $30 \%$ margin on the basis of the frost melting heat, the result is $1007 \mathrm{~kJ}$.

\subsection{According to the structure of the air cooler, determine the ethylene glycol pipeline, calculate the flow resistance of ethylene glycol solution}

The structure of the air cooler selected for this system is shown in Figure 4.

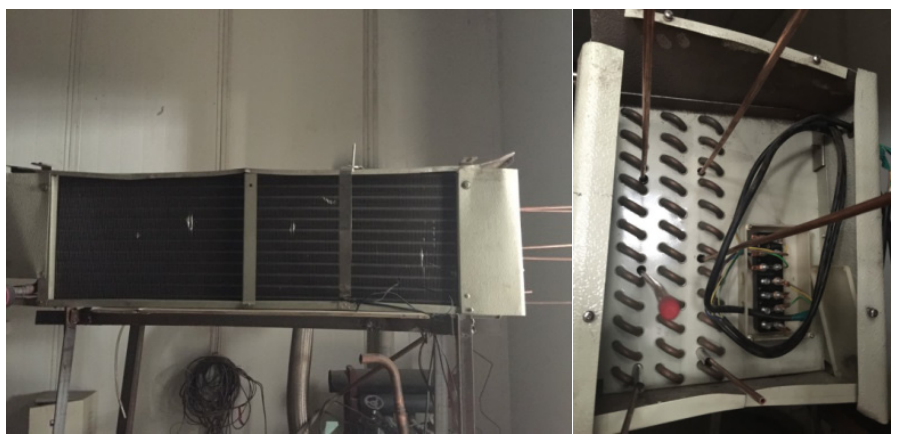

Figure 4. The structure of the air cooler.

According to the size of the air cooler is $1200 \times 400$, ethylene glycol defrosting is arranged on a one-way pipeline, under and out. In order to ensure defrosting, the temperature of ethylene glycol solution entering the air cooler is set at $30^{\circ} \mathrm{C}$, the temperature difference between inlet and outlet is set at $5^{\circ} \mathrm{C}$, and the defrosting time is set at 10 minutes. The circulating amount of ethylene glycol was $0.101 \mathrm{~kg} / \mathrm{s}$.

Selection of ethylene glycol pipe material: considering the heat exchange effect, ethylene glycol pipe is still copper pipe.

The selection of ethylene glycol pipe diameter: in the design of pipe diameter, under the condition of a certain flow, the diameter of the pipe is thicker, the flow resistance is smaller, but the waste of pipe material. On the contrary, the smaller the pipe diameter, the greater the flow resistance, but save space and pipe material. Therefore, when choosing the diameter of the pipe, both should be taken into account. According to the circulating amount of ethylene glycol calculated in the front, the copper tube of DN10 should be preselected.

\section{Technical advantages of waste heat recovery defrost system design}

The project is the first time to carry out the application research on the recovery of compressor exhaust heat in low temperature cold storage. The exhaust temperature of the compressor is relatively high, which has a great impact on refrigerant, lubricating oil and pipeline system. As for this part of heat energy, this project puts forward reasonable utilization for the first time.

The use of ethylene glycol as a heat medium, transported to the side of the air cooler for defrosting, can fill the gap in domestic related technology. In view of the problem of defrosting uniformity and process, the reversible fan is set up to control the rotation of the fan, so that the rotation direction is opposite to that of refrigeration, so as to speed up the defrosting process. Through the matching of refrigeration equipment and the optimization of the system, the performance of the unit can be significantly improved. 


\subsection{Energy conservation and environmental protection}

This system can reduce the energy consumption of small cold storage by $20 \% \sim 30 \%$, reduce emission (equivalent to $\mathrm{CO} 2$ emissions) by $10 \% \sim 20 \%$, and save energy by $10 \% \sim 20 \%$ for medium and large cold storage. The construction investment is equivalent to that of traditional cold storage, and the operation cost can be significantly reduced.

\subsection{Safe and reliable}

This system makes full use of the waste heat of the compressor, solves the hidden danger of fire caused by the temperature control of the electric frost, and the ethylene glycol used is also a non-toxic and harmless organic solvent, which finally realizes the green defrosting. At the same time, the system has little impact on food, no pollution to the food, fully ensure the quality of goods, safe and reliable operation.

\subsection{Wide application prospect}

It is estimated that the defrosting technology developed in this project can save $100,000 \mathrm{kWh}$ of electricity and 120,000 yuan of electricity per year compared with the defrosting technology of electric heating for a 2,000-ton cold storage. The construction investment only increased by more than 50,000 yuan. Moreover, the temperature and pressure of ethylene glycol in the whole cycle are far below the allowable value, so it has a reliable safety guarantee, so it has a broad prospect of popularization and application.

The technical achievements of this project can also be transformed into logistics cold chain, refrigeration and air conditioning, industrial refrigeration and other industrial fields, which will generate greater market demand.

\section{Acknowledgments}

Thanks very much to all the teachers, students of our school refrigeration team and the alumni who have graduated from the enterprise, we can guarantee the normal production of the prototype of waste heat recycling. Thanks to China National Center for Good Operation of Refrigeration for its strong support of this project.

\section{References}

1. Li Lai.(2019) Discussion and design on hot fluor defrosting in cold storage. Refrigerating and AirConditioning, 19:56-58.

2. Zang Runqing, Liu Jianxun, Yuan Bo and Liu Qi.(2015) Comparison study of liquid refrigerant defrosting and electric heating defrosting in refrigeration system. Journal of Thermal Science and Technology, 14:40-44.
3. Liu Enhai, Nan Xiaohong and He Yuan.(2007) Investigation of the Frosting Performance and Improvement of the Defrosting Method for an Air- cooler under Low Temperature Condition. Journal of Refrigeration, 28(2):57-59.

4. Liu Xunhai, Jiang Jingde, Zhu Hua and Zhou Jianjia.(2009) Experimental Comparison of Electricity Defrosting and Hot Gas Defrosting in Cold Storage. Journal of Refrigeration, 30(3):5862.

5. Zhuang Youming.(2006)Discussion and Analysis of Different Defrosting Ways and Their Energy Consumption in Food Cold Storage. Journal of Jimei University, 11(1): 62-65. 